\title{
Theoretical Aspects of Obtaining Pure Inorganic Substances During Crystallization from Solutions
}

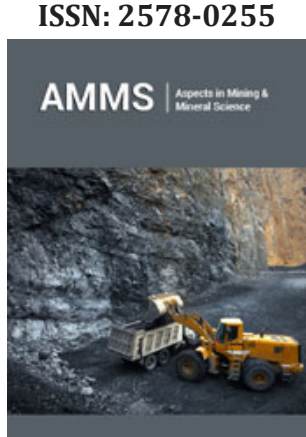

*Corresponding author: GR Allakhverdov, State Scientific-Research Institute of Chemical Reagents and High Purity Chemical Substances-National Research Centre, Kurchatov's Institute, Russia

Submission: 眥July 05, 2019

Published: 侽July 25, 2019

Volume 3 - Issue 2

How to cite this article: Allakhverdov G, Zhdanovich O. Theoretical Aspects of Obtaining Pure Inorganic Substances During Crystallization from Solutions. Aspects Min Miner Sci.3(2). AMMS.000559.2019. DOI: 10.31031/AMMS.2019.03.000559.

Copyright@ GR Allakhverdov, This article is distributed under the terms of the Creative Commons Attribution 4.0 International License, which permits unrestricted use and redistribution provided that the original author and source are credited.

\section{Allakhverdov GR* and Zhdanovich OA}

State Scientific-Research Institute of Chemical Reagents and High Purity Chemical SubstancesNational Research Centre, Kurchatov's Institute, Russia

\begin{abstract}
A method for calculating the cocrystallization of components in ternary water-salt systems is proposed. The method allow to obtain a quantitative assessment of the production of pure inorganic substances in the process of crystallization from electrolyte solutions.
\end{abstract}

Keywords: Cocrystallization coefficient; Activity of component; Isopiestic solution

\section{Introduction}

Crystallization from solutions has long been used as a method of separation and the production of pure substances. This method acquired particular importance in the first half of the last century in the preparation of compounds of radioactive elements, its relevance is preserved today in the technology of high purity substances. Technological developments in this field are based on studies of the properties of solutions whose main task is to evaluate the efficiency of the separation of inorganic compounds during crystallization from electrolyte solutions. In the technology of high purity substances, the problem is reduced to the evaluation of the interaction of the major and individual impurity component, since in field of microconcentrations of impurity components it is possible neglect their interaction with each other.

\section{Theory}

For the ternary water-salt system according to the Nernst distribution law, the thermodynamics coefficient of cocrystallization of the impurity component can be expressed as

$$
D_{2}^{0}=\frac{A_{1} B_{2}}{B_{1} A_{2}}
$$

where A and B activity of components in liquid and solid phases; the subscript 1 and 2 refer to the major and impurity component, respectively. Taking the activity of pure component in the solid phase to be unity, the Eq.(2) can be represented also as $D_{2}^{0}=\frac{A_{1}^{0}}{A_{0}^{0}}$, where $\mathrm{A}_{\mathrm{i}}{ }^{0}$ is the activity component in its saturated binary solution. The activity of each component in the mixed electrolyte solution according to Allakhverdov GR [1] in the first approximation can be represented as

$$
A_{i}=A_{i}^{*} \frac{x_{i}}{\sum x_{i}}
$$

where $A_{i}^{*}$ is the activity of the impurity component in its binary isopiestic solution having the same solvent activity as in the mixed solution; $x=v m / W, v$-stoichiometric coefficient of the electrolyte, $\mathrm{m}$-molality, $\mathrm{W}$ - number of moles of the solvent per kilogram ( $\mathrm{W}=55.51$ for water).

Combining Eqs. (1) and (2) and substitution $\mathrm{B}_{\mathrm{i}}=\mathrm{y}_{\mathrm{i}} \mathrm{f}_{\mathrm{i}}$, where $\mathrm{y}$ and $\mathrm{f}$ is the mole fraction and activity coefficient of a component in the solid phase, we obtain

$$
\frac{A_{1}^{*} x_{1} y_{2} f_{2}}{A^{*}{ }_{2} x_{2} y_{1} f_{1}}=\frac{A_{1}^{0}}{A^{0}{ }_{2}}
$$

In the limiting case when $\mathrm{x}_{2} \rightarrow 0$ also $\mathrm{A}_{1}^{*} \rightarrow \mathrm{A}_{1}^{*}, \mathrm{f}_{1} \rightarrow 1$ and the equilibrium cocrystallization coefficient of the impurity component can be defined as 


$$
D_{2}=\lim _{x_{2} \rightarrow 0} \frac{x_{1} y_{2}}{y_{1} x_{2}}=\frac{1}{f_{2}} \frac{A_{2}^{*}}{A^{0}{ }_{2}}
$$

Relation (4) is similar to the Ratner AP [2], with the distinction that liquid-phase interaction is represented here in explicit form as ratio of the impurity activities in different solutions. In this case $A_{i}^{*}$ is the activity of the impurity component in its binary isopiestic solution having the same solvent activity as in the saturated solution of the major component. If we use the functional dependence of the osmotic coefficient of the solution on the concentration $\varphi=\varphi(\mathrm{x})$, described in [3-5], then the value of $\mathrm{x}$ can be determined from equation: $x \Psi=-\ln a_{w}$, where $\mathrm{a}_{\mathrm{w}}$ is the solvent activity in a saturated solution of major component. Based on the value of $\varphi$, the activity coefficient can be determined and then the activity of impurity component. The activity coefficient of the impurity component in the solid phase $f_{2}$ according to Allakhverdov GR $[6,7]$ can also be calculated based on the data for the pure components.

\section{Conclusion}

Thus, the proposed method makes it possible to predict the change in the concentration of the impurities components during crystallization of the major component from electrolyte solutions..

\section{References}

1. Allakhverdov GR, Mikhlin AL (2017) Separation factor for the crystallization of inorganic substances from aqueous solutions. Inorganic Materials 53(5): 525-528.

2. Ratner AP (1933) On the theory of the distribution of electrolytes between a solid crystalline and liquid phase. J Chem Phys vol.1: 789-794.

3. Allakhverdov GR (2008) Thermodynamics of electrolyte solutions. Doklady Physica 53(8): 420-424.

4. Allakhverdov GR (2012) Coulomb interaction in electrolyte solutions. Doklady Physica 57(6): 221-223.

5. Allakhverdov GR (2014) The conductivity equation for solution of strong electrolytes. Doklady Physica 59(5): 206-208.

6. Urusov VS (1977) Theory of isomorphic mixability. Science Moscow.

7. Allakhverdov GR (2019) Thermodynamics of solutions and separation of elements during crystallization. Moscow. 